\title{
SOME HYPERGEOMETRIC INTEGRALS FOR LINEAR FORMS IN ZETA VALUES
}

\author{
WADIM ZUDILIN
}

(Received 23 April 2018; accepted 12 May 2018; first published online 19 July 2018)

Dedicated to Carlo Viola, whose creativity in and love of integrals for linear forms in zeta values are boundless, on the occasion of his 75th birthday

\section{Abstract}

We prove new integral representations of the approximation forms in zeta values.

2010 Mathematics subject classification: primary 11M06; secondary 11J72, 33C20.

Keywords and phrases: Hurwitz zeta function, hypergeometric integrals, integral representation.

In the exposition below, $s$ and $D$ are positive integers such that $s \geq 3 D-1$, while the parameter $n$ is assumed to be a positive even integer. The notation

$$
\zeta(s, \alpha)=\sum_{n=0}^{\infty} \frac{1}{(n+\alpha)^{s}}
$$

is used for the Hurwitz zeta function, so that $\zeta(s)=\zeta(s, 1)$, and $d_{n}=\operatorname{lcm}(1,2, \ldots, n)$.

In [1] the following approximations are constructed: for any $j \in\{1, \ldots, D\}$, take

$$
r_{n, j}=\sum_{m=1}^{\infty} R_{n}\left(m+\frac{j}{D}\right), \quad \text { where } R_{n}(t)=D^{3 D n} n !^{s+1-3 D} \frac{\prod_{l=0}^{3 D n}(t-n+l / D)}{\prod_{l=0}^{n}(t+l)^{s+1}} .
$$

It is shown that

$$
r_{n, j}=a_{0, j}+\sum_{\substack{2 \leq i \leq s \\ i \equiv s(\bmod 2)}} a_{i} \zeta\left(i, \frac{j}{D}\right)
$$

with

$$
\begin{gathered}
d_{n}^{s+1-i} a_{i} \in \mathbb{Z} \quad \text { for } i=2,3,4, \ldots, s \text { and } i \equiv s(\bmod 2), \\
d_{n+1}^{s+1} a_{0, j} \in \mathbb{Z} \quad \text { for } j \in\{1, \ldots, D\}
\end{gathered}
$$

This is an Open Access article, distributed under the terms of the Creative Commons Attribution licence (http://creativecommons.org/licenses/by/4.0/), which permits unrestricted re-use, distribution, and reproduction in any medium, provided the original work is properly cited.

(c) 2018 Australian Mathematical Publishing Association Inc. 
(see [1, Lemmas 1 and 2]), and some further information is provided for the asymptotic growth of the positive quantities $r_{n, j}$ as $n \rightarrow \infty$. (We note that choosing $n$ even implies that $3 D n+1+(s+1)(n+1) \equiv s(\bmod 2)$ and hence $R_{n}(-n-t)=(-1)^{s} R_{n}(t)$. This reflects on the parity in the summation in (1)—consideration in [1] is restricted to the case of $s$ odd.) The approximations are building blocks for linear forms in zeta values $\zeta(i)$ with $i$ of the same parity as $s$, with the help of the elementary formula

$$
\sum_{j=1}^{d} \zeta\left(i, \frac{j(D / d)}{D}\right)=\sum_{j=1}^{d} \zeta\left(i, \frac{j}{d}\right)=d^{i} \zeta(i)
$$

valid for any divisor $d$ of $D$.

The principal goal of this note is to establish the following integral representation of the approximations $r_{n, j}$ for $j \in\{1, \ldots, D\}$.

THEOREM 1. The linear forms (1) admit the integral representation

$$
r_{n, j}=\frac{D^{s-1}(3 D n+1) !}{n !^{3 D}} \sum_{m=1}^{D} \xi^{-m j} r_{n, m}^{*}
$$

where

$$
r_{n, m}^{*}=\xi^{m} \int_{[0,1]^{s+1}} \ldots \int_{\prod_{i=0}^{s} x_{i}^{D n}\left(1-x_{i}^{D}\right)^{n} d x_{i}}=\int_{0}^{\xi^{m}} \int_{[0,1]^{s}} \ldots \int_{\left.\xi^{m} x_{0} \cdots x_{s}\right)^{3 D n+2}} \frac{\prod_{i=0}^{s} x_{i}^{D n}\left(1-x_{i}^{D}\right)^{n} d x_{i}}{\left(1-x_{0} \cdots x_{s}\right)^{3 D n+2}}
$$

and $\xi=\xi_{D}$ denotes a primitive root of unity of degree $D$.

Proof. As the rational function $R_{n}(t)$ has zeros at $t=m-(D-j) / D$ for $m=1, \ldots, n$ and $j \in\{1, \ldots, D\}$, we can write

$$
\begin{aligned}
r_{n, j}= & \sum_{m=n}^{\infty} R_{n}\left(m+\frac{j}{D}\right)=D^{3 D n} n !^{s+1-3 D} \sum_{k=0}^{\infty} \frac{\prod_{l=0}^{3 D n}(k+(l+j) / D)}{\prod_{l=0}^{n}(k+n+l+j / D)^{s+1}} \\
= & \frac{n !^{s+1-3 D} \prod_{l=0}^{3 D n}(l+j)}{D \prod_{l=0}^{n}(n+l+j / D)^{s+1}} \\
& \left.\times{ }_{s+D+1} F_{s+D}\left|\left\{1+\frac{j-l}{D}: l=1, \ldots, D, j \neq l\right\},\left\{2 n+1+\frac{j}{D}\right\}^{s+1}\right| 1\right) \\
= & \frac{(3 D n+j) !}{D n !^{3 D}(j-1) !} \int_{[0,1]^{s+1}} \ldots \int_{j}\left(t_{0} \cdots t_{s}\right) \prod_{i=0}^{s} t_{i}^{n+j / D-1}\left(1-t_{i}\right)^{n} d t_{i},
\end{aligned}
$$


where

$$
\begin{aligned}
& f_{j}(t)={ }_{D} F_{D-1}\left(\begin{array}{c}
\left\{3 n+\frac{j+l}{D}: l=1, \ldots, D\right\} \\
\left\{1+\frac{j-l}{D}: l=1, \ldots, D, j \neq l\right\}
\end{array} \mid t\right) \\
& =\sum_{k=0}^{\infty} \frac{\prod_{l=1}^{D}\left(3 n+\frac{j+l}{D}\right)_{k}}{\prod_{l=1}^{D}\left(1+\frac{j-l}{D}\right)_{k}} t^{k}=\sum_{k=0}^{\infty} \frac{(3 D n+j+1)_{D k}}{(j)_{D k}} t^{k} \quad \text { for } j \in\{1, \ldots, D\} .
\end{aligned}
$$

Recall that

$$
\sum_{l=0}^{\infty} \frac{(a)_{l}}{l !} x^{l}=\frac{1}{(1-x)^{a}}
$$

and observe that

$$
\begin{aligned}
\frac{(3 D n+2)_{j-1}}{(j-1) !} x^{j-1} f_{j}\left(x^{D}\right) & =\sum_{k=0}^{\infty} \frac{(3 D n+2)_{D k+j-1}}{(D k+j-1) !} x^{D k+j-1} \\
& =\sum_{\substack{l=0 \\
l \equiv j-1(\bmod D)}}^{\infty} \frac{(3 D n+2)_{l}}{l !} x^{l}=\frac{1}{D} \sum_{m=1}^{D} \frac{\xi^{-m(j-1)}}{\left(1-\xi^{m} x\right)^{3 D n+2}}
\end{aligned}
$$

Taking $t_{i}=x_{i}^{D}$ for $i=0,1, \ldots, s$ in the integrals (2), we thus obtain

$$
r_{n, j}=\frac{D^{s-1}(3 D n+1) !}{n !^{3 D}} \sum_{m=1}^{D} \xi^{-m(j-1)} \int_{[0,1]^{s+1}} \ldots \int_{\prod_{i=0}} \frac{\prod_{i}^{D n}\left(1-x_{i}^{D}\right)^{n} d x_{i}}{\left(1-\xi^{m} x_{0} \cdots x_{s}\right)^{3 D n+2}}
$$

for each $j \in\{1, \ldots, D\}$.

Choosing $D=2$ and $s \geq 5$ odd, we obtain the linear forms

$$
\begin{aligned}
7 r_{n, 2}-r_{n, 1}= & \frac{2^{s}(6 n+1) !}{n !^{6}} \int_{[0,1]^{s+1}} \cdots \int_{1}\left(\frac{3}{\left(1-x_{0} x_{1} \cdots x_{s}\right)^{6 n+2}}\right. \\
& \left.-\frac{4}{\left(1+x_{0} x_{1} \cdots x_{s}\right)^{6 n+2}}\right) \prod_{i=0}^{s} x_{i}^{2 n}\left(1-x_{i}^{2}\right)^{n} d x_{i} \\
= & \frac{2^{s}(6 n+1) !}{n !^{6}} \int_{\gamma \times[0,1]^{s}} \ldots \int_{\frac{\prod_{i=0}^{s} x_{i}^{2 n}\left(1-x_{i}^{2}\right)^{n} d x_{i}}{\left(1-x_{0} x_{1} \cdots x_{s}\right)^{6 n+2}}}
\end{aligned}
$$

in $\mathbb{Q}+\mathbb{Q} \zeta(5)+\cdots+\mathbb{Q} \zeta(s)$ considered previously in [2]. Here the path $\gamma \subset \mathbb{R}$ for integrating with respect to $x_{0}$ is given by $\gamma=3[0,1]+4[0,-1]$ and the parity assumption on $n$ can be dropped. 


\section{Acknowledgements}

This note was produced during the trimester on Periods in Number Theory, Algebraic Geometry and Physics at the Hausdorff Research Institute for Mathematics (Bonn, Germany). I thank Clément Dupont for his encouragement to write the integrals for the hypergeometric approximations used in $[1,2]$.

\section{References}

[1] S. Fischler, J. Sprang and W. Zudilin, 'Many odd zeta values are irrational', Preprint, 2018, arXiv:1803.08905 [math.NT]; abridged version, Comptes Rendus Math. Acad. Sci. Paris 356 (2018), 707-711.

[2] W. Zudilin, 'One of the odd zeta values from $\zeta(5)$ to $\zeta(25)$ is irrational. By elementary means', SIGMA 14 (2018), Article ID 028, 8 pages.

WADIM ZUDILIN, Department of Mathematics, IMAPP,

Radboud University, PO Box 9010, 6500 GL Nijmegen, Netherlands

e-mail:w.zudilin@math.ru.nl 